\title{
Lack of influence of non-inherited maternal HLA-DR alleles on susceptibility to rheumatoid arthritis
}

\author{
Alan J Silman, Elaine M Hay, Jane Worthington, Wendy Thomson, Lynne Pepper, \\ Jude Davidson, Phil A Dyer, William E R Ollier
}

\begin{abstract}
Objective-To reproduce findings from previous reports that non-inherited maternal HLA class II antigens might contribute to rheumatoid arthritis (RA) susceptibility in the offspring.

Methods-Families were recruited from the Arthritis and Rheumatism Council's National Repository of RA families and HLA-DRB1 alleles were examined in these individuals and their first degree relatives using DNA typing methods.

Results-There was no evidence of an increase in either non-inherited maternal HLA-DR4 or the HLA-DRB1 shared epitope as a whole compared with the frequency expected using the noninherited paternal antigens as controls. Conclusions-The numbers of probands who were shared epitope negative were small, but we are unable to confirm in these families the findings that noninherited maternal HLA contributes an additional susceptibility factor to rheumatoid arthritis.
\end{abstract}

(Ann Rheum Dis 1995; 54: 311-313)

A strong association between rheumatoid arthritis (RA) and HLA-DR4 antigen has been demonstrated in many population studies. ${ }^{1}{ }^{2}$ Subsequent studies, particularly in nonEuropean populations, have demonstrated associations with other HLA-DR specificities. ${ }^{34}$ With the introduction of DNA based methods to HLA typing, it has become possible to explain these diverse associations of HLA alleles by an association with a conserved sequence of the the third hypervariable region of the DRB1 gene, the so called RA shared epitope. $^{5}$ Even in caucasoid populations, however, an important proportion of RA cases (typically $10-31 \%$ ) are shared epitope negative, ${ }^{67}$ an observation frequently explained on the basis of genetic heterogeneity. An alternative explanation for this lack of consistency was recently proposed, based on the influence in the affected offspring of effects from the non-inherited maternal HLA antigens (conveniently referred to as NIMA). In initial studies, HLA class I NIMA affected the T and B cell repertoire of the offspring in approximately $50 \%$ of individuals, ${ }^{8}$ with evidence of a significant decrease in cytotoxic $\mathrm{T}$ cell activity against the individuals' non-inherited maternal class I antigens. ${ }^{9}$ It was thus of considerable interest that a recent study by the same group demonstrated a threefold increase in the likelihood of RA probands having an NIMA which carried HLA-DR4, compared with a non-inherited paternal antigen (NIPA) which carried HLA-DR4, the latter acting as controls. ${ }^{10}$ This effect was seen to an equal extent in both HLA-DR4 positive and DR4 negative probands. We have examined the possible role of NIMA in a further series of RA families, taking advantage of the greater precision afforded by HLA-DR oligonucleotide typing.

\section{Subjects and methods}

The subjects for this analysis came from the Arthritis and Rheumatism Council's National Repository of RA families. ${ }^{11}$ All affected individuals were interviewed and examined using a standardised protocol, blood was taken for determination of rheumatoid factor (RF), and past data on RF seropositivity and radiological erosions were sought. Only those individuals who satisfied the 1987 American College of Rheumatology criteria ${ }^{12}$ modified for genetic studies ${ }^{13}$ were included; in all, they comprised 74 individuals from 39 families.

DNA was extracted from samples of all these individuals and their first degree relatives and polymerase chain reaction based oligonucleotide typing was undertaken for HLA-DRB1. HLA-DRB ${ }^{\star} 04$ subtyping was undertaken using an amplification refractory mutation system restriction length fragment polymorphism. ${ }^{14}$ For the purposes of this analysis, all samples were categorised as being (i) DR4 positive/negative (to permit comparison with the earlier study) and (ii) shared epitope positive/negative depending on the presence of one of the following alleles: HLA-DRB $1^{\star} 0101$, ${ }^{\star} 0401,{ }^{\star} 0404,{ }^{\star} 0405,{ }^{\star} 0408$. No other DRB $1{ }^{\star} 01$ subtypes were seen in this population. Families were eligible for inclusion in this analysis if they had at least one affected offspring and parental HLA haplotypes could be unambiguously assigned based on the available relatives. Analysis of the segregation of the parental haplotypes in the affected offspring permitted identification of both the inherited and non-inherited haplotypes.

The analysis was undertaken in the following manner. First, the frequencies of NIMA bearing (i) DR4 and (ii) any shared epitope allele were compared with those frequencies in 
Frequencies of HLA-DR4 and HLA-DRB1 shared epitope non-inherited maternal antigens (NIMA) and non-inherited paternal antigens (NIPA) in affected probands

\begin{tabular}{|c|c|c|c|c|}
\hline Proband type & $N I M A$ & $N I P A$ & $\begin{array}{l}\text { Relative risk } \\
(95 \% \text { CI })\end{array}$ & $\begin{array}{l}\text { Weighted relative risk } \\
(95 \% \text { CI })\end{array}$ \\
\hline All & $(n=65)$ & $(n=71)$ & & \\
\hline DR4 +ve & 20 & 30 & $0.73(0.46$ to 1.15$)$ & $0.75(0.42$ to 1.50$)$ \\
\hline DRB1 shared epitope +ve & & 39 & $0.81(0.58$ to 1.15$)$ & $0.87(0.55$ to 1.40$)$ \\
\hline Shared epitope heterozygotes & $(\mathrm{n}=30)$ & $(n=33)$ & & \\
\hline DR4 +ve & 12 & 13 & $1.02(0.56$ to 1.87$)$ & $1 \cdot 12(0.51$ to $2 \cdot 74)$ \\
\hline DRB 1 shared epitope +ve & 14 & 16 & $0.96(0.57$ to 1.62$)$ & $1.06(0.51$ to 2.20$)$ \\
\hline
\end{tabular}

$\mathrm{CI}=$ Confidence interval.

the NIPA of the same probands. The NIPA were used as controls, as adopted in an earlier study. ${ }^{10}$ The results were expressed as relative risks (with $95 \%$ confidence intervals), with the null hypothesis that there would be no greater risk of DR4 shared epitope in NIMA compared with NIPA. Second, given that many of the affected probands were from multicase families, the analysis was repeated by weighting the NIMA and NIPA according to the number of affected offspring in a sibship studied. Thus, if there were two affected siblings, the NIMA and NIPA score was multiplied by 0.5 , so that the total contribution to the overall NIMA and NIPA frequencies from any one mother or father was 1 . Third, the analysis was repeated restricting consideration to those probands who were shared epitope heterozygote on the basis that the homozygotes did not need any involvement from NIMA. There were too few probands who were shared epitope negative for useful separate analysis.

\section{Results}

Data were available from 65 NIMA and 71 NIPA of the 74 probands (table). Of the 39 families, nine had a single affected proband, three had three affected siblings and the remainder had an affected sibling pair. In all, $64(86 \%)$ of the probands were HLA-DR4 positive, of whom 27 were DR4 homozygous. There was no evidence of an increase in NIMA carrying either DR4 or the shared epitope compared with NIPA, in either the weighted or the unweighted analysis. Further, there was no such increase when the analysis was restricted to only those probands who were shared epitope heterozygous. There were too few who were DR4 negative/shared epitope negative for definitive comment, but only one of the NIMAs of the DR4 negative probands carried a DRB $1^{\star} 04$ allele.

\section{Discussion}

Unlike the authors of an earlier report, ${ }^{10}$ we were unable to confirm an increase in DR4bearing alleles in the NIMA of RA probands, whatever the DR4 status of the proband. Given the small numbers of RA probands who were HLA-DR4 negative, it remains possible that, in such patients, DR4-bearing NIMA could contribute to disease susceptibility. There is, however, one major methodological difference between the two studies. The current study utilised the families collected for a multicase family study and the majority of the families had at least two affected siblings. Given the association between RA and HLA-DR, it is hardly surprising that in such families there was a high frequency of DR4. Indeed, it was expected that both the mothers and fathers of such multiple affected sibships would have an increased frequency of these susceptibility alleles. Nevertheless, more than $67 \%$ of the NIMA did not carry HLA-DR4 and more than half were shared epitope negative. These proportions were smaller than those observed in the NIPA collected from the same probands. The hypothesis being addressed was that both inherited and non-inherited maternal alleles are important in leading to disease susceptibility, whereas it is only the inherited paternal ones that are of relevance. There was no evidence from our analysis to support this.

There may, indeed, be genetic heterogeneity, with different genetic contributions to sporadic and 'familial' RA as has been previously proposed. ${ }^{15}$ The only difference reported though, is the increase in DR4 $4^{16}$ which would be expected from the known association between HLA and RA. Others have found no such excess in familial disease. ${ }^{17}$ Thus it seems unlikely that the failure to find an effect of NIMA in these families is a result of the relative lack of importance of HLA in explaining disease susceptibility. The converse is more likely, given the high level of homozygosity in these cases.

We acknowledge the financial support of the Arthritis and Rheumatism Council who established the Repository with a special project grant. The ARC Repository also receives support from the European Union's Concerted Action on Immunotherapy. We are most grateful to our rheumatology colleagues for notifying us of families and providing clinical data.

1 Stastny P. Association of the B cell alloantigen DRw4 with rheumatoid arthritis. $N$ Engl f Med 1978; 298: 869-71.

Ollier W, Thomson W. Population genetics of rheumatoid arthritis. Rheum Dis Clin N Am 1992; 18: 741-59.

3 Woodrow J C, Nichol F E, Zaphiropoulos G. DR antigens and rheumatoid arthritis: a study of two populations. and rheumatoid arthritis:

4 Wilkens R F, Hansen J A, Malmgren J A, et al. HLA antigens in Yakima Indians with rheumatoid arthritis. Arthritis Rheum 1982; 25: 1435-9.

5 Gregersen P K, Silver J, Winchester R J. The shared epitope hypothesis. Arthritis Rheum 1987; 30: 1205-13.

6 Wordsworth P, Pile K D, Buckely J D, et al. HLA heterozygosity contributes to susceptibility to rheumatoid arthritis. Am $\mathcal{F}$ Hum Genet 1992; 51: 585-91.

7 Jawaheer D, Thomson W, MacGregor A J, et al. "Homozygosity" for the HLA-DR shared epitope contributes the highest risk for rheumatoid arthritis concordance in identical twins. Arthritis Rheum 1994; 37 681-6.

8 Claas F H J, Gijbels Y, Van der Velden-de Munck J, van Rood J J. Induction of B cell unresponsiveness to noninherited maternal HLA antigens during fetal life. Science 1988; 241: 1815-7.

9 Zhang L, Li S G L, van Rood J J, Claas F H J. The T cell repertoire is not dictated by self antigens alone. Res repertoire is not dictated by

10 Ten Wolde S, Breedveld F C, De Vries R R P, et al. Influence of non-inherited maternal HLA antigens on occurrence of rheumatoid arthritis. Lancet 1993; 341: 200-2. 
11 Worthington J, Ollier W E R, Leach M K, et al. The Arthritis and Rheumatism Council's National Repository of Family Material: pedigrees from the first $100 \mathrm{RA}$ families containing affected sibling pairs. Br $\mathcal{F}$ Rheumatol 1994; 33 970-6.

12 Arnett F C, Edworthy S M, Bloch D A, et al. The American Rheumatism Association 1987 Revised Criteria for the classification of rheumatoid arthritis. Arthritis Rheum 1988; 31: 315-24.

13 MacGregor A J, Bamber S, Silman A J. A comparison of the performance of different methods of disease lassification for rheumatoid arthritis. Results of an analysis from a nationwide twin study. $\mathrm{Br} f$ Rheumato
14 Jawaheer D, Ollier W E R, Thomson W. Multiplex ARMS RFLP: a simple and rapid method for HLA-DR4 subtyping. Eur f Immunogen 1993; 20: 175-87.

15 Sanders P A, Grennan D M, Dyer P A, Thomson W, deLange GG. A comparison of clinical and immunogenetic features in familial and sporadic rheumatoid arthritis. $\mathcal{F}$ Rheumatol 1987; 14: 718-22.

16 Wolfe F. Age of death of parents of patients with rheumatoid arthritis: data from a middle class sample. 7 Rheumatol 1989; 16: 735-9.

17 Deighton C M, Walker D J. What factors distinguish probands from multicase rheumatoid arthritis same sex sibships from sporadic disease? Br $\mathcal{J}$ Rheumatol 1992; 19: 237-41. 